\title{
The prognostic equation for biogeochemical tracers . has no unique solution.
}

Corresponding author: J.F. Geurdes, Geurdes datascience kvk64522202, C vd Lijnstraat 164, 2593 NN, the Hague, Netherlands (han.geurdes@gmail.com) 
3 Abstract. In this paper, a tracer prognostic differential equation related

4 to the marine chemistry HAMOCC model is studied. Recently the present

5 author found that the Navier - Stokes equation has no unique general solu-

${ }_{6}$ tion [Geurdes, 2017]. The following question can therefore be justified. Do

7 numerical solutions, found from prognostic equations akin to the Navier Stokes

s equation, provide unique nutrient distribution information. 


\section{Introduction.}

9 Recent insights into global biogeochemical cycles, such as the key limiting role of iron ${ }_{10}$ as a nutrient [Martin and Fitzwater, 1988], justify a computational model search for the

${ }_{11}$ distribution of iron in the Oceans. Other elements like phosphorus also play a key role.

${ }_{12}$ Both iron as well as phosphorus influence the growth of phytoplankton. In addition, ${ }_{13}$ factors such as light are, obviously, important [Popova, Ryabchenko and Fasham, 2000].

${ }_{14}$ In the present paper, we concentrate on chemical tracers that can be described in a 15 prognostic equation. The prognostic is considered the kernel of numerical computations.

${ }_{16}$ This equation is also to be found in studies on marine aggregates [Liu, Kindler and Khalili, ${ }_{17}$ 2012], their equation (10).

18 One thing that catches the eye when looking at prognostic equations for tracers is 19 that their mathematical structure resembles somewhat the Navier Stokes equation (NSE).

${ }_{20}$ There are numerical models such as HAMOCC [Ilynia, Six, Segschneider, Maier-Reimer, ${ }_{21}^{21}$ Li and Nunez-Riboni, 2013] based on those kernel equations. We note that in the field of ${ }_{22}$ biogeochemical cycle study, numerical studies based on the prognostic equations are sup${ }_{23}$ posed to fill in the gaps where data is sparse [Manizza, Follows, Dutkiewicz, McClelland, ${ }_{24}$ Menemenlis, Hill, Townsend-Small and Peterson, 2009].

${ }_{25}$ Recently, the present author [Geurdes, 2017] showed that the NSE in three dimensions ${ }_{26}$ does not have a general solution. Such a conclusion leaves room for so called weak solu${ }_{27}$ tions. However, the weak solutions and their numerical equivalents are most likely not ${ }_{28}$ unique in the absence of an exact solution. Therefore it is methodologically interesting to 29 turn the attention to tracer prognostics equations as a "NSE next of kin" equation. We 
so are, therefore, allowed to ask if models based on that type of equation can have unique

${ }_{31}$ numerical solutions. If there is no unique solution associated to such an equation, the

32 validation of the global cycle models perhaps do not allow the conclusions attached to

з3 them. Most likely more empirical validation via nutrient sampling is then necessary. A

${ }_{34}$ similar case can be made for $\mathrm{CO}_{2}$ concentration models based on the prognostic.

\section{Mathematical model prognostic.}

35 The prognostic equation used by Dutkiewicz, Follows and Parekh [2005] can be written 36 as,

37

$$
\frac{\partial A(x)}{\partial t}+\nabla \cdot(\mathbf{u}(x) A(x))-\nabla \cdot(\mathbf{K}(x) \nabla A(x))=S_{A}(x)
$$

${ }_{38}$ This equation is the kernel of the HAMOCC model equation. Accordingly, we refer to 39 equation (1) in Ilynia, Six, Segschneider, Maier-Reimer, Li and Nunez-Riboni [2013]. In 40 our equation (1), the abbreviation, $x=(\mathbf{x}, t) \in \mathbb{R}^{3} \times \mathbb{R}^{+}$, represents the space and time ${ }_{41}$ coordinates. Let us take $t$ in a finite interval $[0, T]$ with $0<T<\infty$. Furthermore, ${ }_{42} \mathbf{u}(x)$, is the "transformed mean Eulerian" circulation and $\mathbf{K}(x)$ the mixing tensor. $S_{A}(x)$ ${ }_{43}$ describes the sources and sinks of the tracer, which concentration in (1) is denoted by ${ }_{44} A(x)$.

\subsection{Approximate interval}

${ }_{45}$ In this sub section we look at an approximation which appears to be valid given the ${ }_{46}$ context of oceanic bio-cycle studies. Let us concentrate on a certain fully connected finite ${ }_{47}$ space and time set $\Omega \subset \mathbb{R} \times \mathbb{R} \times \mathbb{R} \times \mathbb{R}^{+}$. Suppose, $\mathbf{x}=\left(x_{1}, x_{2}, x_{3}\right)$ and $t \in[0, T]$, then

${ }_{48} \Omega$ is the cartesian product of intervals $\Omega=\left[x_{1,0}, x_{1,1}\right] \times\left[x_{2,0}, x_{2,1}\right] \times\left[x_{3,0}, x_{3,1}\right] \times\left[t_{0}, t_{1}\right]$.

49 Here, $x_{m, 0}<x_{m, 1}$, for $m=1,2,3$, and $t_{0}<t_{1}$. Now for, $x \in \Omega$ we may approximate the 
${ }_{50}$ tracer equation in $(1)$ with $\mathbf{u}(x) \approx \mathbf{u}^{0}$ a constant vector in $\Omega$ approximating the Eulerian

${ }_{51}$ circulation in $\Omega$. Subsequently, $\mathbf{K}(x) \approx \mathbf{K}^{0}$ the approximate constant mixing tensor and

${ }_{52}$ approximate constant source-sink function $S_{A}(x) \approx S_{A}^{0}$. Those approximate constant $\mathbf{u}, \mathbf{K}$

${ }_{53}$ and $S_{A}$ are reasonable given the physical problem at hand. We also suppose symmetrical

${ }_{54}$ mixing $K_{m, j}^{0}=K_{j, m}^{0}$. Subsequently, $A^{0}=A-t S_{A}^{0}$, allows the prognostic equation to be

55 written like

$$
\frac{\partial A^{0}(x)}{\partial t}+\left(\mathbf{u}^{0} \cdot \nabla\right) A^{0}(x)-\nabla \cdot\left(\mathbf{K}^{0} \nabla A^{0}(x)\right)=0
$$

${ }_{57}$ Here the right hand approximation is replaced with an equal sign for ease of notation. Let

${ }_{58}$ us, further, introduce a spectral parameter breakdown of the solution such as in [Quispel,

${ }_{59}$ 1983]. E.g. we can have a (real) measure $\nu$ and real parameter $k \in \mathbb{R}$ such that

$$
A^{0}(x)=\int_{\mathbb{R}} d \nu(k) A_{k}^{0}(x)
$$

${ }_{61}$ Subsequently let us look at functions carrying a spectral parameter, $k$. One would cer-

${ }_{62}$ tainly agree that if we find two different functions, $A_{1, k}^{0}(x)$ and $A_{2, k}^{0}(x)$ that solve $(2)$,

${ }_{63}$ then, via the integration over the measure $\nu$ in $(3)$, two different functions $A_{1}^{0}(x)$ and ${ }_{64} A_{2}^{0}(x)$ are obtained.

${ }_{65}$ Before showing such two function let us start by introducing the plane wave [Quispel,

${ }_{66}$ 1983], factor for $x \in \Omega$,

67

$$
\rho_{k, \mathbf{b}}(x)=\exp \left[-\|\mathbf{b}\|^{2} k^{2} t-i k(\mathbf{b} \cdot \mathbf{L x})\right]
$$

${ }_{68}$ In the definition of the plane wave the $\|\mathbf{b}\|^{2}$ represents the Euclidean norm of the constants ${ }_{69}$ vector $\mathbf{b}$. Furthermore, $\mathbf{L}$ is a real $3 \times 3$ constant-in-x tensor (matrix) such that, $p=$ $70 \quad 1,2,3, n=1,2,3$

$$
\mathbf{b} \cdot \mathbf{L x}=\sum_{p} b_{p} \sum_{n} L_{p, n} x_{n}
$$


72 Hence we find that, for $m=1,2,3$,

$$
\frac{\partial}{\partial x_{m}}(\mathbf{b} \cdot \mathbf{L x})=\sum_{p} b_{p} L_{p, m}
$$

\subsection{Integral equation}

Starting from $x \in \Omega$, it is possible to write down an integral equation that resembles the ones that are employed for linearizion of nonlinear partial differential equations [Nijhoff, Quispel, van der Linden and Capel, 1983]

$$
A_{1, k}^{0}(x)+i A_{2, k}^{0}(x)=\sum_{q} r_{q, k} \rho_{i q, \mathbf{a}}(x)+i \sum_{q} s_{q, k} \rho_{i q, \mathbf{b}}(x)
$$

78 In this equation, $q \in \mathbb{Z}^{+}-\{0\}$, together with, $r_{q, k} \in \mathbb{C}$ and, $s_{q, k} \in \mathbb{R}$. Note that $\mathbf{a}$ and $\mathbf{b}$

79 are different vectors. Moreover, the $A_{2, k}^{0}(x)$ in equation (7) is defined by

$$
A_{2, k}^{0}(x)=\iint_{\mathbb{R}^{2}} d \lambda(\ell) d \lambda\left(\ell^{\prime}\right) \frac{\rho_{\ell-\ell^{\prime}, \mathbf{b}}(x)}{\ell+\ell^{\prime}} A_{1, k+\ell+\ell^{\prime}}^{0}(x)
$$

${ }_{81}$ Hence, equation(7) is an integral equation comparable to what is employed by Quispel ${ }_{82}$ [1983]. In the equation (8) the $\lambda$ is a real measure and $\ell$ and $\ell^{\prime}$ are real numbers. If, for ${ }_{83}$ arbitrary $k$, we have $A_{1, k}^{0}(x) \in \mathbb{R}$ then it follows, because of symmetry in exchange of $\ell$ ${ }_{84}$ and $\ell^{\prime}$, while noting $\left\{\rho_{\ell-\ell^{\prime}, \mathbf{b}}(x)\right\}^{*}=\rho_{-\ell+\ell^{\prime}, \mathbf{b}}(x)$ and $*$ is complex conjugation, that $A_{2, k}^{0}(x)$ ${ }_{85}$ is a real number too. Moreover, it is noted that if a real (domain and co-domain) operator ${ }_{86} \mathcal{O}$ exists such that the right-hand side of the integral equation (5) vanishes, then we find ${ }_{87}$ that, $\mathcal{O} A_{1, k}^{0}(x)+i \mathcal{O} A_{2, k}^{0}(x)=0$. If $\mathcal{O} A_{j, k}^{0}(x) \in \mathbb{R}$, for $j=1,2$, then if $\mathcal{O} A_{1, k}^{0}(x) \in \mathbb{R}$ and ${ }_{88} \mathcal{O} A_{2, k}^{0}(x) \in \mathbb{R}$ it follows that, $\mathcal{O} A_{1, k}^{0}(x)=\mathcal{O} A_{2, k}^{0}(x)=0$.

\subsection{Operations and parameters for linearization.}

\subsubsection{The $\mathrm{u}^{0} \cdot \nabla$ operator}


HAN GEURDES: NAVIER STOKES TYPE OF TRACER PROGNOSTIC EQUATIONS. $\quad \mathrm{X}-7$

90 Let us start the investigation of the approximate prognostic by noting that the operation

${ }_{91} \quad \mathbf{u}^{0} \cdot \nabla$ can be written in a sum format $\sum_{m} u_{m}^{0} \frac{\partial}{\partial x_{m}}$, with $m=1,2,3$. Hence applying this

${ }_{92}$ operation to the left and right hand side of the integral equation in (7) we find

93

$$
\begin{array}{r}
\sum_{m} u_{m}^{0} \frac{\partial A_{1, k}^{0}(x)}{\partial x_{m}}+i \sum_{m} u_{m}^{0} \frac{\partial A_{2, k}^{0}(x)}{\partial x_{m}}= \\
\sum_{m} u_{m}^{0} \frac{\partial}{\partial x_{m}}\left[\sum_{q} r_{q, k} \rho_{i q, \mathbf{a}}(x)+i \sum_{q} s_{q, k} \rho_{i q, \mathbf{b}}(x)\right]
\end{array}
$$

${ }_{95}$ If we for the moment write $\mathbf{y}$ for $\mathbf{a}$ or $\mathbf{b}$ it can be acknowledged that

$$
\frac{\partial}{\partial x_{m}} \rho_{i q, \mathbf{y}}(x)=q \rho_{i q, \mathbf{y}}(x) \frac{\partial}{\partial x_{m}}(\mathbf{y} \cdot \mathbf{L x})
$$


With the use of the expression in (6) it follows

$$
\frac{\partial}{\partial x_{m}} \rho_{i q, \mathbf{y}}(x)=q \rho_{i q, \mathbf{y}}(x) \sum_{p} y_{p} L_{p, m}
$$

99

100 ${ }_{113}$ look at

114

Returning to equation (8) and bearing in mind the above exercise with the operator $\mathbf{u}^{0} \cdot \nabla$, it can be concluded that $\mathbf{u}^{0} \cdot \nabla$ is a $\mathcal{O}$-type operator aluded to in section-2.2. Hence, $\mathbf{u}^{0} \cdot \nabla A_{1, k}^{0}(x)=\mathbf{u}^{0} \cdot \nabla A_{2, k}^{0}(x)=0$.

\subsubsection{The $\frac{\partial}{\partial t}-\nabla \cdot\left(K^{0} \nabla\right)$ operator}

With the result of section-2.3.1 in mind let us turn the attention to the rest of the operator employed in the approximate prognostic equation. The operator $\frac{\partial}{\partial t}-\nabla \cdot\left(\mathbf{K}^{0} \nabla\right)$ is complicated. Let us start by looking at $\frac{\partial}{\partial t}$. This operation is applied to the right hand side of the integral equation (7). The tactics of how to approach the operator $\frac{\partial}{\partial t}$ on the right hand side of the integral equation resembles that of the section-2.3.1. So we may

$$
\frac{\partial}{\partial t} \rho_{i q, \mathbf{y}}(x)=\|\mathbf{y}\|^{2} q^{2} \rho_{i q, \mathbf{y}}(x)
$$

Applying the operator $\nabla \cdot\left(\mathbf{K}^{0} \nabla\right)$ to the right hand of the integral equation (7) implies

$$
\begin{array}{r}
\sum_{m, j} \frac{\partial}{\partial x_{m}} K_{m, j}^{0} \frac{\partial}{\partial x_{j}} \rho_{i q, \mathbf{y}}(x)=q \sum_{m, j} \frac{\partial}{\partial x_{m}} \rho_{i q, \mathbf{y}}(x) K_{m, j}^{0} \sum_{p} y_{p} L_{p, j}= \\
q^{2} \rho_{i q, \mathbf{y}}(x) \sum_{m, j} K_{m, j}^{0} \sum_{p^{\prime}} y_{p^{\prime}} L_{p^{\prime}, m} \sum_{p} y_{p} L_{p, j}
\end{array}
$$


Subsequent reshuffling of the sums gives

$$
\begin{aligned}
& \sum_{m, j} \frac{\partial}{\partial x_{m}} K_{m, j}^{0} \frac{\partial}{\partial x_{j}} \rho_{i q, \mathbf{y}}(x)=q^{2} \rho_{i q, \mathbf{y}}(x) \sum_{p^{\prime}} \sum_{p} y_{p^{\prime}} y_{p} \sum_{m, j} K_{m, j}^{0} L_{p^{\prime}, m} L_{p, j}= \\
& q^{2} \rho_{i q, \mathbf{y}}(x) \sum_{p^{\prime}} \sum_{p} y_{p^{\prime}} y_{p} \delta_{p, p^{\prime}}=q^{2} \rho_{i q, \mathbf{y}}(x)\|\mathbf{y}\|^{2}
\end{aligned}
$$

Here, the $\mathbf{L}$ matrix is such that

$$
\sum_{m, j} K_{m, j}^{0} L_{p^{\prime}, m} L_{p, j}=\delta_{p, p^{\prime}}
$$

This is the condition for (15), $m, j, p, p^{\prime}=1,2,3$ and $q \in \mathbb{Z}^{+}-\{0\}$. Subsequently, looking at (13) and the result of (15) then

$$
\left(\frac{\partial}{\partial t}-\nabla \cdot\left(\mathbf{K}^{0} \nabla\right)\right) \rho_{i q, \mathbf{y}}(x)=0
$$

Hence, the operator $\frac{\partial}{\partial t}-\nabla \cdot\left(\mathbf{K}^{0} \nabla\right)$ is also of the type of $\mathcal{O}$ alluded to in section-2.2. Looking at the integral equation (7) this implies that

$$
\left(\frac{\partial}{\partial t}-\nabla \cdot\left(\mathbf{K}^{0} \nabla\right)\right) A_{n, k}^{0}(x)=0
$$

and here, $n=1,2$. Hence, combining this result with $\mathbf{u}^{0} \cdot \nabla A_{1, k}^{0}(x)=\mathbf{u}^{0} \cdot \nabla A_{2, k}^{0}(x)=0$, it must be possible for the approximate prognostic equation to have two solutions at least. One solution is derived from $A_{1, k}^{0}(x)$ the other from $A_{2, k}^{0}(x)$, which in fact is an integral transformation, given in (8), of $A_{1, k}^{0}(x)$.

\subsection{Consistency for $A_{2, k}^{0}$ under $\frac{\partial}{\partial t}-\nabla \cdot\left(K^{0} \nabla\right)$}

33 In order to study the consistency of the claim, we need to look at the transformation in (8). The two $x$ dependent terms are of the greatest importance in the evaluation.

Therefore let us introduce the abbreviation $\langle\cdot\rangle$ to denote the weighted integration in the transformation (8). So,

$$
A_{2, k}^{0}(x)=\left\langle\rho_{\ell-\ell^{\prime}, \mathbf{b}}(x) A_{1, g}^{0}(x)\right\rangle
$$



with the abbreviation, $g=k+\ell+\ell^{\prime}$. Looking at the operator form we find

$$
\frac{\partial}{\partial t} A_{2, k}^{0}(x)=\left\langle-\left(\ell-\ell^{\prime}\right)^{2}\|\mathbf{b}\|^{2} \rho_{\ell-\ell^{\prime}, \mathbf{b}}(x) A_{1, g}^{0}(x)\right\rangle+\left\langle\rho_{\ell-\ell^{\prime}, \mathbf{b}}(x) \frac{\partial}{\partial t} A_{1, g}^{0}(x)\right\rangle
$$

140

141

142

$$
\left\langle\sum_{m, j} \frac{\partial}{\partial x_{m}} K_{m, j}^{0} \rho_{\ell-\ell^{\prime}, \mathbf{b}}(x)\left\{(-i)\left(\ell-\ell^{\prime}\right) \sum_{p} b_{p} L_{p, j} A_{1, g}^{0}(x)+\frac{\partial}{\partial x_{j}} A_{1, g}^{0}(x)\right\}\right\rangle
$$

143 Subsequently, the $\frac{\partial}{\partial x_{m}}$ gives, suppressing for the moment, the $x$ dependence in the notation

144

$$
\begin{array}{r}
{ }_{145}\left\langle\rho_{\ell-\ell^{\prime}, \mathbf{b}} \sum_{m, j} K_{m, j}^{0}(-i)\left(\ell-\ell^{\prime}\right) \sum_{p^{\prime}} b_{p^{\prime}} L_{p^{\prime}, m}\right. \\
\left.{ }_{146} \quad\left\langle(-i)\left(\ell-\ell^{\prime}\right) \sum_{p} b_{p} L_{p, j} A_{1, g}^{0}+\frac{\partial A_{1, g}^{0}}{\partial x_{j}}\right\}\right\rangle+ \\
\left\langle\rho_{\ell-\ell^{\prime}, \mathbf{b}} \sum_{m, j} K_{m, j}^{0}\left\{(-i)\left(\ell-\ell^{\prime}\right) \sum_{p} b_{p} L_{p, j} \frac{\partial A_{1, g}^{0}}{\partial x_{m}}+\frac{\partial^{2} A_{1, g}^{0}}{\partial x_{j} \partial x_{m}}\right\}\right\rangle
\end{array}
$$

$$
W_{1}=\left\langle(-1)\left(\ell-\ell^{\prime}\right)^{2}\|\mathbf{b}\|^{2} \rho_{\ell-\ell^{\prime}, \mathbf{b}}(x) A_{1, g}^{0}(x)\right\rangle
$$

$$
W_{3}=\left\langle\rho_{\ell-\ell^{\prime}, \mathbf{b}}(x) \nabla \cdot\left(\mathbf{K}^{0} \nabla\right) A_{1 g}^{0}(x)\right\rangle
$$

${ }_{154}$ The previous three forms allow the following conclusion

${ }_{155}\left(\frac{\partial}{\partial t}-\nabla \cdot\left(\mathbf{K}^{0} \nabla\right)\right) A_{2, k}^{0}(x)=2 i\left\langle\rho_{\ell-\ell^{\prime}, \mathbf{b}}(x) \sum_{m, j} K_{m, j}^{0}\left\{\left(\ell-\ell^{\prime}\right) \sum_{p} b_{p} L_{p, j} \frac{\partial A_{1, g}^{0}(x)}{\partial x_{m}}\right\}\right\rangle(26)$ 

170 that

171 Hence,

$$
\begin{array}{r}
\sum_{m, j, p} b_{p} K_{m, j}^{0} L_{p, j} \frac{\partial}{\partial x_{m}} A_{1, k}^{0}(x)+i \sum_{m, j, p} b_{p} K_{m, j}^{0} L_{p, j} \frac{\partial}{\partial x_{m}} A_{2, k}^{0}(x)= \\
\sum_{m, j, p} b_{p} K_{m, j}^{0} L_{p, j} \frac{\partial}{\partial x_{m}}\left(\sum_{q} r_{q, k} \rho_{i q, \mathbf{a}}(x)+i \sum_{q} s_{q, k} \rho_{i q, \mathbf{b}}(x)\right)
\end{array}
$$

\subsubsection{The operator $\sum_{m, j, p} b_{p} K_{m, j}^{0} L_{p, j} \frac{\partial}{\partial x_{m}}$.}

The result in $(27)$ and therefore the consistency, $\left(\frac{\partial}{\partial t}-\nabla \cdot\left(\mathbf{K}^{0} \nabla\right)\right) A_{2, k}^{0}(x)=0$ can be studied by applying the operator, $\sum_{m, j, p} b_{p} K_{m, j}^{0} L_{p, j} \frac{\partial}{\partial x_{m}}$ to the integral equation in (7).

Similar to e.g. section-2.3.1, the use of $\rho_{i q, \mathbf{y}}(x)$ is justified looking at (28). So, it follows that, ignoring the $q$ sums and coefficients for the moment,

$$
\sum_{m, j, p} b_{p} K_{m, j}^{0} L_{p, j} \frac{\partial}{\partial x_{m}} \rho_{i q, \mathbf{y}}(x)=q \rho_{i q, \mathbf{y}}(x) \sum_{m, j, p} b_{p} K_{m, j}^{0} L_{p, j} \sum_{p^{\prime}} y_{p^{\prime}} L_{p^{\prime}, m}
$$

In this equation we may recognize, $\sum_{m, j} K_{m, j}^{0} L_{p^{\prime}, m} L_{p, j}=\delta_{p, p^{\prime}}$ from (16). This implies

$$
\sum_{m, j, p} b_{p} K_{m, j}^{0} L_{p, j} \frac{\partial}{\partial x_{m}} \rho_{i q, \mathbf{y}}(x)=q \rho_{i q, \mathbf{y}}(x) \sum_{p} b_{p} y_{p}
$$

We note again that $\mathbf{y}=\mathbf{a}$ resembles the right hand side of (28). Hence if $\mathbf{a}$ and $\mathbf{b}$ are orthogonal, the first term in (28) on the right hand side vanishes. Then, because we work with real functions and coefficients, it follows that

$$
\sum_{m, j, p} b_{p} K_{m, j}^{0} L_{p, j} \frac{\partial}{\partial x_{m}} A_{1, k}^{0}(x)=0
$$


${ }_{176}$ If this is combined with the result from (27) the consistency, $\left(\frac{\partial}{\partial t}-\nabla \cdot\left(\mathbf{K}^{0} \nabla\right)\right) A_{2, k}^{0}(x)=0$ 177 applies. Comparing the imaginary parts on left and right hand of (28) it is also found 178 that, using $\mathbf{y}=\mathbf{b}$ in (30), the consistency requires that

$$
\sum_{m, j, p} b_{p} K_{m, j}^{0} L_{p, j} \frac{\partial}{\partial x_{m}} A_{2, k}^{0}(x)=\sum_{q} q\|\mathbf{b}\|^{2} s_{q, k} \rho_{i q, \mathbf{b}}(x)
$$

$$
\iint_{\mathbb{R}^{2}} d \lambda(\ell) d \lambda\left(\ell^{\prime}\right)(-i)\left(\ell-\ell^{\prime}\right) \frac{\rho_{\ell-\ell^{\prime}, \mathbf{b}}(x)}{\ell+\ell^{\prime}} A_{1, k+\ell+\ell^{\prime}}^{0}(x)=\sum_{q} q s_{q, k} \rho_{i q, \mathbf{b}}(x)
$$

Here equation (16) is employed to the result of the $\frac{\partial}{\partial x_{m}}$ differentiation on the left hand side, such that the $\|\mathbf{b}\|^{2}$ drops off. It can be easily verified, taking the complex conjugate and interchanging the $\ell$ and the $\ell^{\prime}$, that the left hand of (33) is real. So, this requirement can be met in principle for $x \in \Omega$. We also note that from $\left(\frac{\partial}{\partial t}-\nabla \cdot\left(\mathbf{K}^{0} \nabla\right)\right) \rho_{i q, \mathbf{y}}(x)=0$ in (17) and from equation (31) together with $\left(\frac{\partial}{\partial t}-\nabla \cdot\left(\mathbf{K}^{0} \nabla\right)\right) A_{1, k}^{0}(x)=0$, the equation in (33) is consistent.

\section{Conclusion}

It was demonstrated that the solution of an approximation of the tracer prognostic equation of Dutkiewicz, Follows and Parekh [2005] has no unique solution. This agrees with another finding that the Navier Stokes equation (NSE) in $d=3$ spatial dimensions has no general exact solution [Geurdes, 2017]. The form of the prognostic of e.g. Dutkiewicz, Follows and Parekh [2005] resembles the NSE.

The question can therefore be raised what the numerical solution of [Dutkiewicz, Follows and Parekh, 2005] is telling us. 

approach.

Of course it has to be acknowledged that the infinite sequence that can be derived from the prognostic equation is approximative. The marine biogeochemistry, however, seems to allow the approximation. Moreover, it also has to be acknowledged that the present infinite sequence of solutions is not tested with numerical analysis. Our argument uncovers to a principle. Numerical studies will not take the principle objection of a multitude of different solutions, away.

Subsequently, we have to acknowledge that the infinite series of solutions has a particular form. The operators $\mathbf{u}^{0} \cdot \nabla$ and $\frac{\partial}{\partial t}-\nabla \cdot\left(\mathbf{K}^{0} \nabla\right)$ working on the start-point solution, $A^{0}=A_{1}^{0}$, must identically vanish. We note that if $A^{0} \approx A-t S_{A}^{0}$, this is an approximate solution to the prognostic for the domain $x \in \Omega$. Of course, if it is argued that only the $A$ 's with $\left(\mathbf{u}^{0} \cdot \nabla\right) A=\left(\frac{\partial}{\partial t}-\nabla \cdot\left(\mathbf{K}^{0} \nabla\right)\right) A=0$, show the infinite transformation characteristic, then one already agrees that the prognostic has not got a unique solution. Then the question can rightfully be raised why the numerical outcomes of the model computations are not checked for uniqueness.

Another point to raise is that the obtained infinite series of solutions all are bounded by a certain finite time interval. Hence, a possible test of correctness of HAMOCC numerical computations could be to check the numerical solution at "infinite time". It can be true that stable behavior at infinite time also implies uniqueness. However, the fact that the NSE does not have a general exact solution [Geurdes, 2017] raises doubt about such an

The final question then is how the result of this paper affects the conclusions of HAMOCC-like model computations. It appears to make sense to claim that when there is no unique solution to the kernel equation of the model, then the verification of validity of 
${ }_{219}$ the model computations cannot come from numeric model computations such as claimed ${ }_{220}$ by $[$ Manizza, Follows, Dutkiewicz, McClelland, Menemenlis, Hill, Townsend-Small and ${ }_{221}$ Peterson, 2009].

The subsequent question might then be: How bad is the result of this mathematical exercise for model computations? Here we will answer this question conceptually and ${ }_{224}$ pose the real question. We have demonstrated that

225

$$
A_{1, k}^{0}(x)+i A_{2, k}^{0}(x)=\sum_{q} r_{q, k} \rho_{i q, \mathbf{a}}(x)+i \sum_{q} s_{q, k} \rho_{i q, \mathbf{b}}(x)
$$

226 contains two different forms of solution to the same prognostic differential equation. This equation is key to the HAMOCC model. To be more specific

$$
A_{2, k}^{0}(x)=\iint_{\mathbb{R}^{2}} d \lambda(\ell) d \lambda\left(\ell^{\prime}\right) \frac{\rho_{\ell-\ell^{\prime}, \mathbf{b}}(x)}{\ell+\ell^{\prime}} A_{1, k+\ell+\ell^{\prime}}^{0}(x)
$$

${ }_{229}$ is a spectral integral transformation of $A_{1, k}^{0}(x)$. It implies that if one has one (numerical) ${ }_{230}$ solution, another one can be obtained. From that one, yet another one can be obtained etc, ${ }_{231}$ etc. The single question we ask is. How can the reseachers that use model computations ${ }_{232}$ of nutrients distribution in the ocean, be certain that a consistent "filling the gaps of ${ }_{233}$ measurement" [Manizza, Follows, Dutkiewicz, McClelland, Menemenlis, Hill, Townsend${ }_{234}$ Small and Peterson, 2009] description is obtained? here

\section{References}

Dutkiewicz, S., M.J. Follows and P. Parekh, Interactions of the iron and phospho-

${ }_{236}$ rus cycles: A three-dimensional model study, Global Biogeochemistry Cycles, 19, doi:10.1029/2004GB002342, 2005. 
Geurdes, H, A type D breakdown of the Navier Stokes equation in d=3 spatial dimensions, Cogent Mathematics 4, 1284293, doi:10.1080/23311835.2017.1284293, 2017.

Ilyina,T., K.D. Six, J. Segschneider, E. Maier-Reimer, H. Li and I. Nunez-Riboni, Global ocean biogeochemistry model HAMOCC, J. Adv. Mod. Earth Syst, 5, 286-315, 2013.

Liu, B, K. Kindlre, A. Khalili, Dynamic solute release from marine aggregates, Limnol. Oceanogr. Fluids \& Environment 2, 109-120, 2012, doi 10.1215/21573689-2016772.

Nijhoff, F.W., G.R.W. Quispel, J. van der Linden and H.W. Capel, On some linear integral equations generating solutions of non-linear partial differential equations, Physica 119A, 101-142, 1983.

Manizza, M., M.J. Follows, S. Dutkiewicz, J.W. McClelland, D. Menemenlis, C.N. Hill, A. Townsend-Small, B.J. Peterson, Modeling transport and fate of riverine dissolved organic carbon in the Artic Ocean, Global Biogeochemistry Cycles, 23, doi:10.1029/2008GB003396, 2009.

Martine, J.H. and S. Fitzwater, Iron deficiency limits phytoplankton growth in the northeast Pacific subarctic, Nature, 331, 341-343, 1988.

Popova, E.E., V.A. Ryabchenko and M.J.R. Fasham, Biological pump and vertical mixing in the Southern Ocean: Their impact on atmospheric $\mathrm{CO}_{2}$, Global Biogeochemistry Cycles, 14, 477-498, 2000.

Quispel, G.R.W. Linear integral equations and soliton systems, PhD thesis Leiden, 3-5, 1983. 